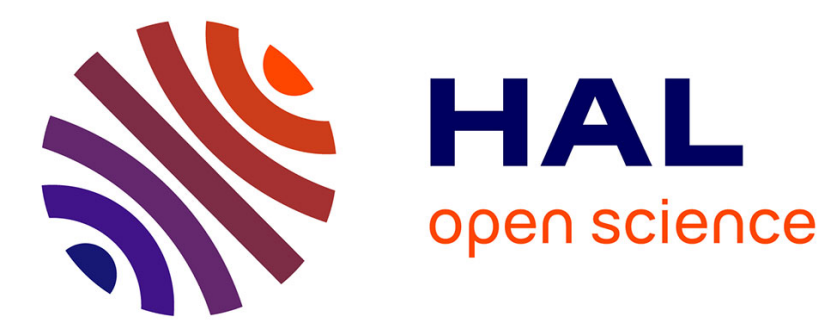

\title{
Endonucleolytic RNA cleavage by a eukaryotic exosome
}

\author{
Alice Lebreton, Rafal Tomecki, Andrzej Dziembowski, Bertrand Séraphin
}

\section{To cite this version:}

Alice Lebreton, Rafal Tomecki, Andrzej Dziembowski, Bertrand Séraphin. Endonucleolytic RNA cleavage by a eukaryotic exosome. Nature, 2008, 456 (7224), pp.993-6. 10.1038/nature07480 . hal01350769

\section{HAL Id: hal-01350769 \\ https://hal-ens.archives-ouvertes.fr/hal-01350769}

Submitted on 1 Aug 2016

HAL is a multi-disciplinary open access archive for the deposit and dissemination of scientific research documents, whether they are published or not. The documents may come from teaching and research institutions in France or abroad, or from public or private research centers.
L'archive ouverte pluridisciplinaire HAL, est destinée au dépôt et à la diffusion de documents scientifiques de niveau recherche, publiés ou non, émanant des établissements d'enseignement et de recherche français ou étrangers, des laboratoires publics ou privés. 


\title{
Endonucleolytic RNA cleavage by a eukaryotic exosome
}

Alice Lebreton $^{1,2,3, *}$ Rafal Tomecki ${ }^{4,5 *}$, Andrzej Dziembowski ${ }^{4,5, \$} \&$ Bertrand Séraphin $^{1,2,3, \sharp}$

Received 14 May; accepted 25 September 2008, published online 7 December 2008.

1. Équipe Labellisée La Ligue, Centre de Génétique Moléculaire, CNRS UPR 2167, 91198 Gif-surYvette, France;

2. Université Paris-Sud, 91405 Orsay, France;

3. Université Pierre et Marie Curie-Paris 6, 75005;

4. Department of Genetics and Biotechnology, Faculty of Biology, University of Warsaw, 02-106 Warsaw, Poland;

5. Institute of Biochemistry and Biophysics, Polish Academy of Sciences, 02-106 Warsaw, Poland.

$\ddagger$ Correspondence and requests for materials should be addressed to A.D. (andrzejd@ibb.waw.pl) or B.S. (seraphin@igbmc.fr).

This is an accepted manuscript of an article published in Nature by Macmilian Publishers, Part of Springer Nature, on 18/12/2008. Reprints and permissions information is available at http://www.nature.com/reprints. (C) Macmillan Publishers. The published article is available online: http://www.nature.com/nature/journal/v456/n7224/full/nature07480.html.

\begin{abstract}
The exosome is a major eukaryotic nuclease located in both the nucleus and the cytoplasm that contributes to the processing, quality control and/or turnover of a large number of cellular RNAs ${ }^{1-6}$. This large macromolecular assembly has been described as a $3^{\prime} \rightarrow 5^{\prime}$ exonuclease ${ }^{1}$ and shown to contain a nine-subunit ring structure evolutionarily related to archaeal exosome-like complexes and bacterial polynucleotide phosphorylases. Recent results have shown that, unlike its prokaryotic counterparts, the yeast and human ring structures are catalytically inactive. In contrast, the exonucleolytic activity of the yeast exosome core was shown to be mediated by the RNB domain of the eukaryote-specific Dis3 subunit $^{7-9}$. Here we show, using in vitro assays, that yeast Dis3 has an additional endoribonuclease activity mediated by the PIN domain located at the amino terminus of this multidomain protein. Simultaneous inactivation of the endonucleolytic and exonucleolytic activities of the exosome core generates a synthetic growth phenotype in vivo, supporting a physiological function for the PIN domain. This activity is responsible for the cleavage of some natural exosome substrates, independently of exonucleolytic degradation. In contrast with current models, our results show that eukaryotic exosome cores have both endonucleolytic and exonucleolytic activities, mediated by two distinct domains of the Dis 3 subunit. The mode of action of eukaryotic exosome cores in RNA processing and degradation should be reconsidered, taking into account the cooperation between its multiple ribonucleolytic activities.
\end{abstract}


The exosome complex, first identified as a $3^{\prime} \rightarrow 5^{\prime}$ exonuclease necessary for the 3'-' end processing of the yeast $5.8 \mathrm{~S}$ ribosomal $\mathrm{RNA}^{1}$, was later implicated in the 3'-5' end processing of many stable RNA species ${ }^{2}$, in messenger RNA turnover ${ }^{3}$, in various mRNA surveillance pathways ${ }^{4}$ and in the degradation of cryptic unstable transcripts (CUTs) ${ }^{5}$. The exosome is therefore a major eukaryotic 3 ' $\rightarrow$ 5' exonuclease with pleiotropic functions ${ }^{6}$.

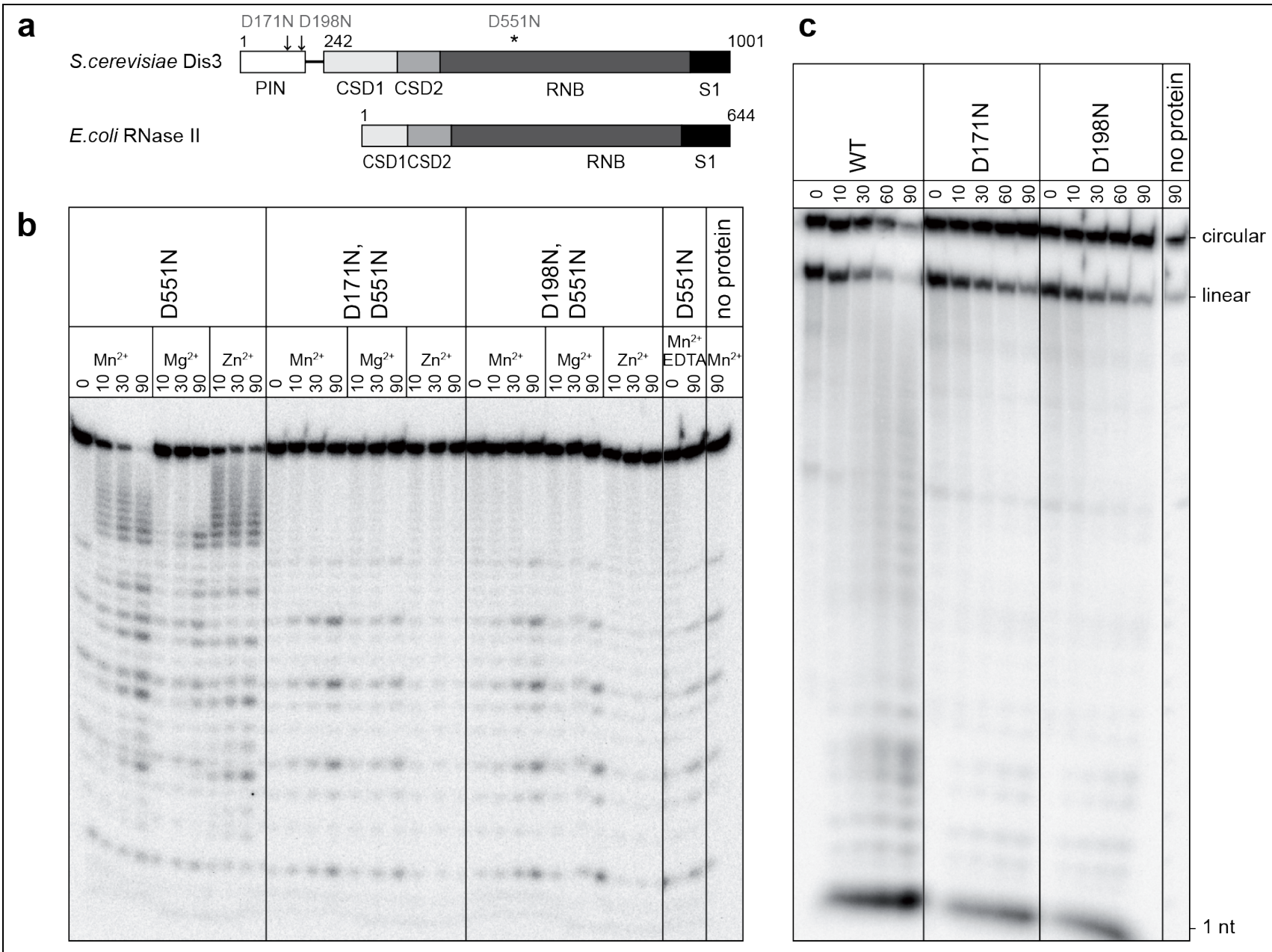

Figure 1 I Endonucleolytic activity of the Dis3 PIN domain in vitro. a, Domain organization and amino-acid numbering of yeast Dis3 and E. coli RNase II: RNB domains have exoribonucleolytic activity, OB-folds (CSD1, CSD2 and S1) are RNA-binding domains, and the N-terminal PIN domain is unique to Dis3. Sites mutated in this study are indicated. b, Mutations of conserved aspartates of the Dis3 PIN domain abolish its endoribonucleolytic activity. Gel-electrophoresis analysis of products generated by the incubation of the 5'-labelled 17- $\mathrm{A}_{14}$ oligoribonucleotide (5'-P; 3'-OH) with various Dis3 versions, in the presence of various divalent cations, for the durations indicated (minutes). Preferential cleavage at adenine residues was observed for this particular substrate but was not investigated further. c, The Dis3 PIN domain degrades circular RNA substrates. Wild-type (WT) Dis3 or PIN-domain mutants were incubated with a circularized oligoribonucleotide in the presence of $3 \mathrm{mM} \mathrm{MnCl}$ for the durations indicated (minutes). Linear substrate molecules result from spontaneous cleavage or breakage during handling before the reaction.

Eukaryotic exosomes consist of a conserved core interacting with nuclear or cytoplasmic specific subunits, and pathway-specific cofactors. The core consists of a ring structure formed by nine subunits $^{9,10}$, similar to the archaeal exosome and bacterial phosphorolytic nucleases, and the Dis 3 protein (also called Rrp44), which contains a catalytic RNB domain responsible for the activity of 
hydrolytic RNase II/R family members ${ }^{11-13}$. Association of Dis3 with the ring structure seems stable in yeast but may be more labile in other species ${ }^{6}$. Recent studies indicated that eukaryotic ring structures are devoid of nuclease activity and that only the Dis3 RNB domain is a functionally active $3^{\prime} \rightarrow 5^{\prime}$ exonuclease $e^{7,8}$. In yeast, the non-essential $3^{\prime} \rightarrow 5^{\prime}$ exoribonuclease nuclear-specific subunit Rrp6 cooperates with Dis $3^{14}$.

In addition to the RNB domain and three OB-fold nucleic-acid-binding domains present in all RNase II/R family members ${ }^{11-13}$, Dis3 harbours a highly conserved N-terminal PIN domain (Fig. 1a and Supplementary Fig. 1). PIN domains have been shown to adopt a RNase H fold, some of which are endowed with nuclease activity ${ }^{15,16}$. An alignment of Dis3 PIN domain sequences with those of PIN domains of known structure reveals that all acidic residues essential for the coordination of divalent cations, and thus putatively involved in catalysis, are conserved (Supplementary Fig. 1). To test whether the N-terminal PIN domain of Dis3 is endowed with ribonucleolytic activity, we assayed RNA degradation by Dis $3_{\mathrm{D} 551 \mathrm{~N}}$, a recombinant Dis3 mutant of the RNB domain devoid of exoribonucleolytic activity ${ }^{7}$. Dis $3_{\text {D551N }}$ slowly degraded a short 5'-end-labelled oligoribonucleotide $17-\mathrm{A}_{14}\left(\right.$ CCCCACCACCAUCACUUA $\left.{ }_{14}\right)$ in a divalent-cation-dependent manner $\left(\mathrm{Mg}^{2+}, \mathrm{Mn}^{2+}\right.$ or $\mathrm{Zn}^{2+}$; Fig. 1b). Higher concentrations of divalent cations (1-3 mM) were required than were optimal for exoribonucleolytic activity ${ }^{7}$. To determine whether the PIN domain was responsible for the activity observed with Dis $3_{\text {D551N }}$, we purified mutant proteins (Dis $3_{\text {D171N,D551N }}$ and Dis $3_{\text {D198N,D551N }}$ ) with additional single asparagine substitutions of conserved aspartic residues in the predicted PINdomain catalytic centre (Fig. 1a and Supplementary Fig. 1). We obtained comparable quantities of the single-mutant and double-mutant proteins with equivalent degrees of purity (Supplementary Fig. 2a). The ability of Dis $3_{\text {D551N }}$ to degrade the 5'-labelled RNA substrate in the presence of divalent cations was lost in the double mutants (Fig. 1b), showing that this RNase activity requires an intact Dis3 PIN domain. In addition, recombinant PIN domain alone (Dis3 amino-acid residues 1-241), but not D171N or D198N mutants of this domain, was able to degrade the same RNA substrate (Supplementary Fig. 3), showing that the PIN activity acts independently of the other Dis3 domains. The Dis $3_{\text {D551N }}$ activity was highest with $\mathrm{Mn}^{2+}$ and lowest with $\mathrm{Mg}^{2+}$, in good agreement with results obtained for another PIN domain ${ }^{16}$. Degradation intermediates observed with the 5'-end-labelled substrate were consistent with either a $3^{\prime} \rightarrow 5^{\prime}$ exonucleolytic activity or an endonucleolytic one. The degradation of a 3'-end-labelled substrate by Dis $3_{\mathrm{D} 551 \mathrm{~N}}$, but not by the double mutants, revealed a complementary profile of intermediates (Supplementary Fig. 4), indicating that the activity was most probably endonucleolytic.

Comparison of the activities of the wild-type protein with single and double mutants of the PIN and RNB domains at different concentrations of divalent cations demonstrated endonucleolytic degradation in the context of an exonucleolytically active protein and indicated cooperation of the two activities (Supplementary Fig. 5). Moreover, the wild-type protein, but not the mutants of the PIN domain (Dis $3_{\mathrm{D} 171 \mathrm{~N}}$ or Dis $3_{\mathrm{D} 198 \mathrm{~N}}$ ), was able to digest a circular RNA molecule (Fig. 1c). Taken together, these results show that the Dis3 PIN domain is responsible for a previously unidentified endoribonucleolytic activity of the yeast exosome. The Dis3 protein therefore possesses two distinct nuclease domains that cooperate for RNA degradation, and their respective contributions in vitro depend on the assay conditions.

To test the physiological relevance of the PIN activity, we introduced wild-type or mutated dis 3 versions, fused to the protein A tag, on centromeric plasmids in a strain in which the essential chromosomal DIS3 copy was under the control of a doxycyclin-repressible promoter ${ }^{17}$. We tested the growth phenotypes in the presence or absence of doxycyclin at various temperatures (Fig. 2). After repression of the wild-type DIS3 allele, the D171N PIN mutant showed a modest growth defect at elevated temperatures. Growth of the exonucleolytically inactive mutant D551N was impaired, as reported ${ }^{7}$. Finally, Dis $3_{\text {D171N,D551N }}$ was much more profoundly affected than each single mutant. Mutant phenotypes did not result from protein instability or inability to assemble into 
exosome cores (Supplementary Fig. 6). As the growth phenotype paralleled the relative nuclease activities observed in vitro (Supplementary Fig. 5), this result indicates that both nuclease activities of Dis3 are physiologically relevant. The D171N mutation also exacerbated the growth phenotype of the $\operatorname{rrp} 6 D$ mutant, further supporting the functional implication of PIN in exosome function (Supplementary Fig. 7). In the absence of doxycyclin, the DIS $3_{D I 7 I N, D 55 I N}$ allele generated a weak dominant-negative phenotype at $37^{\circ} \mathrm{C}$, indicating that this inactive protein is expressed and probably blocks exosome function.

Figure 2 | Synthetic cell-growth phenotypes of PIN mutants. Growth phenotypes resulting from expression of plasmid-borne DIS3, $D I S 3_{D 55 I N}, D I S 3_{D I 7 I N, D 55 I N}$ or $D I S 3_{\text {DI7IN }}$ alleles were assessed in the presence (repressed chromosomal DIS3) or absence (expressed chromosomal DIS3) of doxycyclin (DOX) after incubation for $50 \mathrm{~h}$ at 25 or $37^{\circ} \mathrm{C}$. -LEU, without leucine.

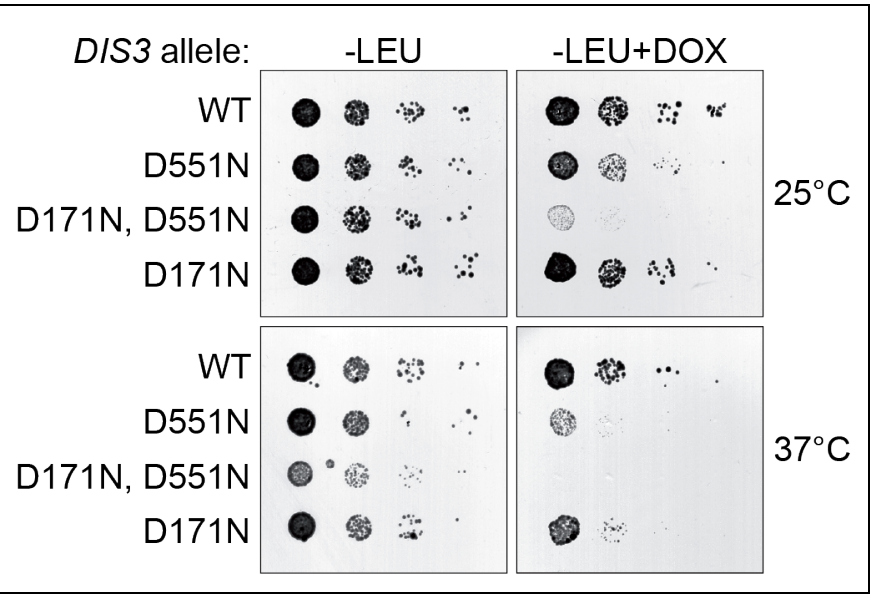

Analysis of total RNA by northern blotting with an oligonucleotide probe complementary to the 3' end of the 5'-external transcribed spacer (ETS) by-product of rRNA processing, a known exosome substrate ${ }^{2}$, revealed that both the D171N and D551N mutants accumulated this species, with a stronger effect for $d i s 3_{D 551 N}$ (Fig. 3a, top panel). The double mutant accumulated even higher amounts of this RNA, its levels reaching those observed in the absence of Dis3. A series of discrete 5'-ETS degradation intermediates was detected in the D551N mutant. These species were no longer visible for dis $3_{D 171 N, D 55 I N}$, suggesting that the PIN endonucleolytic activity was involved in their generation.

Further hybridizations performed with oligonucleotide or randomly primed probes spanning the 5'-ETS region revealed that the intermediates originated from various regions of this RNA, suggesting that they do not result from a simple $5^{\prime} \rightarrow 3^{\prime}$ or $3^{\prime} \rightarrow 5^{\prime}$ exonucleolytic decay (Supplementary Fig. 8). Deletion of RRP6 had modest effects on the degradation pattern, indicating that 5'-ETS digestion depends essentially on both endonuclease and exonuclease activity of Dis3. We used primer extension and circularization RT-PCR (cRT-PCR ${ }^{18,19}$ ) to identify precisely the 5', and 3' ends of 5'-ETS intermediates accumulating in the Dis $3_{\text {D551N }}$ mutant (Fig. $3 b$ and Supplementary Fig. 9a). Intermediates, with sizes ranging from 79 to 462 nucleotides, spanned the complete 5'-ETS. Some of them contained short non-templated 3'-terminal heterogeneous oligo(A) tails, probably synthesized by the TRAMP (Trf4/Air2/Mtr4p polyadenylation) complex, a known exosome stimulator ${ }^{5,20-22}$. More importantly, the 3' end of some species precisely abutted the 5' end of other molecules, which is further evidence of their endonucleolytic origin (Fig. 3b). 5'-ETS cleavages formed in vivo mapped preferentially to internal and closing loops as well as to other single-stranded regions (Supplementary Fig. 9b), making an endonucleolytic rather than an exonucleolytic origin more likely. Similar 5'-ETS cleavages could be reproduced in vitro (Fig. 3c; compare with intermediate sizes observed with the random-primed probe shown in Supplementary Fig. 8).

Northern blot analyses targeted to other natural exosome substrates showed that the PIN domain endonuclease activity was not specific for the 5'-ETS (Fig. 3a, lower panels). D171N and D551N mutations in Dis3 had synergistic effects on the degradation of the CUT NEL025 and on the processing of the $7 \mathrm{~S}$ precursor into $5.8 \mathrm{~S}$ rRNA. These effects were further enhanced in an $r r p 6 D$ 
strain (data not shown). The endonucleolytic activity of the exosome core therefore targets a wide range of physiological RNA species, indicating that its role is general.
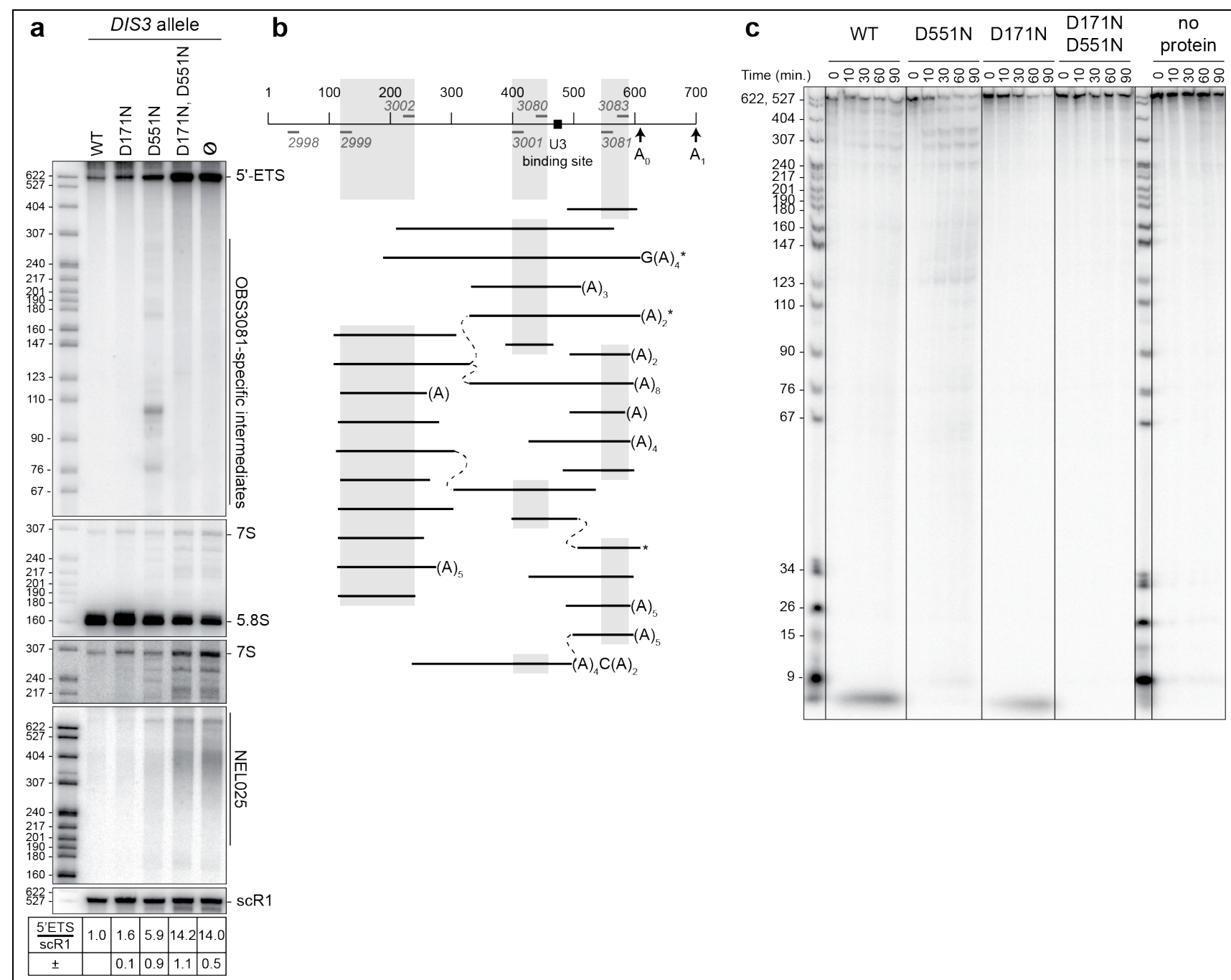

Figure 3 | Dis3 PIN domain mediates cleavage of natural exosome substrates in vivo. a, Detection by northern blot of 5'-ETS, 5.8S rRNA and the NEL025 CUT in total RNA isolated from wild-type and mutant Dis3 strains. The top blot was probed with OBS3081 recognizing the 3' of 5'ETS, the middle blots with a probe complementary to the 5.8S rRNA (two exposures), and the bottom blot with a random-primed probe overlapping the NEL025 CUT. Sizes in nucleotides of the denatured DNA ladder is specified on the left. 0 designates the empty vector. The values in the table represent the ratio of full-length 5'-ETS to control scR1 levels (means \pm s.d.). b, cRT-PCR mapping of 5'-ETS intermediates accumulating in a dis $3_{D 55 I N}$ mutant strain. A bar indicates each intermediate identified, with grey areas highlighting the region required for PCR amplification by the selected primer pairs numbered in italics. Asterisks indicate fragments ending at $\mathrm{A}_{0}$. Dashed lines link 3' ends to 5' ends of contiguous fragments. Polyadenylated 3' ends are indicated between parentheses. c, In vitro cleavage of 5'-ETS by Dis3. 5'-ETS substrate was prepared by in vitro transcription and incubated alone or with various Dis3 versions for the durations indicated. Products resulting from endonucleolytic cleavages were separated by gel electrophoresis from short oligoribonucleotides resulting from exoribonucleolytic degradation that migrated at the bottom of the gel.

Our results show that the yeast exosome, and probably exosomes from other eukaryotes, have endonuclease activity in addition to their long-known exonucleolytic activity. The current model of exosome action should therefore be revisited, because these observations have direct implications 
for the mechanism of recruitment and degradation of substrates. Inactivation of the Dis3 PIN domain by point mutation has limited effects on RNA processing and cell growth. This suggests that RNAs are still degraded in the absence of endonucleolytic cleavages, but probably more slowly or inefficiently. The PIN domain therefore seems to cooperate with the exonucleolytic activity of Dis3 and/or Rrp6, possibly by providing them with alternative 3'-end substrates, which is consistent with the additive phenotypes seen in multiple mutants. This feature could facilitate the degradation of structured RNA species by allowing degradation reinitiation in upstream regions when exosome progression is blocked. Dis3 is therefore a unique example of a RNase in which endoribonucleolytic and exoribonucleolytic catalytic sites are found encoded by different domains of a single protein. Given the structure of related bacterial and archaeal enzymes, Dis3 is likely to originate from the fusion of a PIN domain to a RNase II/R enzyme during the early steps of eukaryotic evolution. In Escherichia coli, the main complex involved in RNA degradation, the degradosome, also has both endonucleolytic and exonucleolytic activities, even though the functions are performed by proteins with no similarity to Dis3, namely endoribonuclease $\mathrm{E}$ and polynucleotide phosphorylase ${ }^{23}$. This evolutionary convergence supports the functional importance for cooperation between exonucleolytic and endonucleolytic activities. Further analysis should help us to understand the relative contributions and spatial arrangements of the two active sites of core eukaryotic exosomes.

Supplementary Information is linked to the online version of the paper at http://www.nature.com/nature/journal/v456/n7224/suppinfo/nature07480.html.

Acknowledgements We thank E. Conti and J. Basquin for providing the Dis3 PIN domain expression construct and $6 \times$ His-SUMO protease, and E. Conti, E. Lorentzen, J. Kufel and members of our groups for insightful discussions. This work was supported by La Ligue contre le Cancer (Équipe Labellisée 2008), Agence Nationale de la Recherche project CUTs, CNRS, ESF RNA Quality program (project EUxosome) and the FP6 EU grant 3D repertoire, and an EMBO installation grant. R.T. is the recipient of the Stipend for Young Researchers from the Foundation for Polish Science and was supported through a Faculty of Biology, University of Warsaw intramural grant.

Author Contributions R.T. expressed and purified the recombinant proteins and performed all the in vitro experiments under the supervision of A.D. A.L. performed all in vivo experiments under the supervision of B.S. All authors discussed the results and wrote the paper. 


\section{Methods}

\section{RNase assays}

In vitro enzymatic assays were performed in $20-\mu l$ reaction volumes containing $10 \mathrm{mM}$ Tris- $\mathrm{HCl}$ $\mathrm{pH} 8.0,75 \mathrm{mM} \mathrm{NaCl}$, and $1 \mathrm{mM}$ 2-mercaptoethanol, with $\mathrm{MnCl}_{2}$ and/or $\mathrm{MgCl}_{2}$ or $\mathrm{ZnCl}_{2}$ at final concentrations ranging from 0 to $3 \mathrm{mM}$. Purified protein and substrate concentrations were 0.1 and $0.2 \mu \mathrm{M}$, respectively. Reaction mixtures were incubated at $30{ }^{\circ} \mathrm{C}$ for the indicated durations before the addition of 1 volume of formamide loading dye (90\% formamide, $20 \mathrm{mM}$ EDTA, 0.03\% bromophenol blue, $0.03 \%$ xylene cyanol in $1 \times \mathrm{TBE}$ ). Reaction products were resolved in denaturing gels containing $20 \%$ polyacrylamide, $8 \mathrm{M}$ urea and $1 \times \mathrm{TBE}$, and were detected with a Fuji PhosphorImager.

\section{cRT-PCR}

The circularization RT-PCR (cRT-PCR) procedure, adapted from previous studies ${ }^{18,19}$, permitted the mapping of 5' and 3' ends in non-polyadenylated, non-capped RNA intermediates. Total RNA from the D551N mutant strain BSY1668[pBS3270] was treated with DNase I. RNA (7 mg) was ligated in the presence of $1 \mathrm{ml}$ of T4 RNA ligase for $2 \mathrm{~h}$ at $37{ }^{\circ} \mathrm{C}$ in a $100-\mathrm{ml}$ final reaction volume. The circularized RNAs were extracted with phenol-chloroform, precipitated with ethanolammonium acetate, and resuspended in $20 \mathrm{ml}$ of water. Reverse transcriptions were performed on $600 \mathrm{ng}$ of circularized RNA with $10 \mathrm{pmol}$ of primers OBS2999, OBS3001 or OBS3081 (Supplementary Table 1) and $1 \mathrm{ml}$ of iScript reverse transcriptase (Bio-Rad). The cDNA junctions were then amplified by PCR with the cognate primer pair: either OBS2999-OBS3002 or OBS3001-OBS3080 or OBS3081-OBS3083. Products were cloned into pCR2.1-TOPO by TOPOTA cloning (Invitrogen), and sequenced with the M13 reverse primer.

\section{Oligonucleotides, plasmids, bacterial strains and yeast strains}

Oligonucleotides used in this work are described in Supplementary Table 1, plasmids in Supplementary Table 2 and yeast strains in Supplementary Table 3.

Plasmids pAD260 and pAD262 were generated by site-directed mutagenesis with oligonucleotides RTADZ-32 to RTADZ-33, using pAD258 or pAD259 as templates, respectively. pAD261 and pAD263 were obtained by site-directed mutagenesis with oligonucleotides RTADZ34 to RTADZ-35, employing pAD258 or pAD259 as templates. D171N and D198N mutations were confirmed by PCR with primers T7-long to RTADZ-10, followed by digestion with TaqI or BshTI, respectively. pAD264 expression vector, encoding the $\mathrm{N}$-terminal fusion of wild-type PIN domain with $6 \times$ His-SUMOTag was constructed by cloning the sequence corresponding to residues 1-241 of Dis3 into BamHI and XhoI sites of pET-28M-SUMO Kan vector. pAD265, pAD266 and pAD267 were obtained by site-directed mutagenesis with oligonucleotides RTADZ-30 to RTADZ-31, RTADZ-32 to RTADZ-33 and RTADZ-34 to RTADZ-35, respectively, and pAD264 as a template. D171N and D198N mutations were confirmed as above, and D91N mutation in pAD265 was verified by PCR with primers T7-long to RTADZ-10, followed by digestion with DraI. pAD268 was generated by cloning of a PCR product corresponding to the 5 -ETS, made with RTADZ-70 to RTADZ-71 primers using genomic DNA from BMA64 strain as template, into pCR-Blunt II-TOPO.

pBS3211 and pBS3210 were obtained by TOPO-blunt cloning (Invitrogen) of PCR products made with oligonucleotides OBS2638 and OBS2639 on DNA from yeast strains BSY1704 and BSY1708, respectively. The insert contained part of the LYS2 sequence, the complete DIS3 or dis $3_{D 55 I N}$ open reading frame in fusion with a tobacco etch virus (TEV) protease cleavage siteProtein A (PA) tag, and the TRP1 marker. The D171N mutation was introduced in these plasmids 
by site-directed mutagenesis with OBS2648 and OBS2649, giving pBS3241 and pBS3245, respectively. The four DIS3 alleles, together with their promoter sequences and the TEV-PA tag, were excised from the TOPO vectors by digestion with SpeI, and cloned into the yeast LEU2 centromeric plasmid pFL36 ${ }^{24}$, giving pBS3269 to pBS3278. pBS3318 was obtained by TOPO-TA cloning of a PCR product corresponding to the 5'-ETS amplified with oligonucleotides OBS2616 and CS18 from genomic DNA.

For expression of the various DIS3 alleles, BSY1668, BSY1865 and BSY1883 were transformed with plasmids pBS3269 to pBS3278. Cultures in synthetic complete medium without leucine were grown at $28^{\circ} \mathrm{C}$. The chromosomal, wild-type DIS3 allele was repressed by the addition of doxycyclin $\left(20 \mu \mathrm{g} \mathrm{ml}^{-1}\right)$.

\section{Heterologous expression and purification of recombinant proteins}

For purification of full-length Dis3 versions, E. coli BL21-CodonPlus-RIL strain (Stratagene) was transformed with the appropriate plasmid (pAD258 to pAD263). Selected transformants were grown in 11 of selective medium after inoculation from a pre-culture at $D_{600} \approx 0.1$. Growth was performed at $37^{\circ} \mathrm{C}$ until $D_{600}$ reached $0.45-0.5$. Cultures were cooled to $16^{\circ} \mathrm{C}$ for $30 \mathrm{~min}$. Protein expression was induced by the addition of isopropyl $\beta$-D-thiogalactoside to $0.5 \mathrm{mM}$ and incubation was continued overnight at $16^{\circ} \mathrm{C}$. After centrifugation, bacterial pellets were resuspended in buffer A (20 mM Tris- $\mathrm{HCl}$ pH 8.0, $200 \mathrm{mM} \mathrm{NaCl}, 10 \mathrm{mM}$ imidazole, $10 \mathrm{mM}$ 2-mercaptoethanol) and lysed by sonication, followed by ultracentrifugation. Soluble fractions were loaded on a His-Trap FF Crude column on an Äkta FPLC purification system (GE Healthcare). The column was washed with buffer A and subsequently with buffer B $(20 \mathrm{mM}$ Tris- $\mathrm{HCl} \mathrm{pH} 8.0 ; 1 \mathrm{M} \mathrm{NaCl}, 10 \mathrm{mM}$ imidazole, $10 \mathrm{mM}$ 2-mercaptoethanol). After re-equilibration with buffer A, bound proteins were eluted with buffer $\mathrm{C}(20 \mathrm{mM}$ Tris- $\mathrm{HCl} \mathrm{pH} 8.0,200 \mathrm{mM} \mathrm{NaCl}, 600 \mathrm{mM}$ imidazole, $10 \mathrm{mM}$ 2mercaptoethanol). Proteins were further purified by ion-exchange chromatography with a MiniQ 4.6/50 PE column followed by size-exclusion chromatography on a Superdex 200 10/300 GL column (both from GE Healthcare). Final elution was performed in buffer containing $10 \mathrm{mM}$ Tris$\mathrm{HCl} \mathrm{pH} 8.0,150 \mathrm{mM} \mathrm{NaCl}$. Finally, glycerol was added to the protein preparations (30\% final concentration), which were stored at $-80{ }^{\circ} \mathrm{C}$. The purity and quantity of the proteins were estimated by analysis in NuPAGE gels (Invitrogen).

Various PIN versions were purified as follows. Transformation of E. coli BL21-CodonPlus-RIL with expression vectors (pAD264 to pAD267; see Supplementary Table 2), culture conditions, the induction of protein expression and extract preparation procedures were the same as for the fulllength Dis3 proteins (see above). Initial purification of $6 \times$ His-SUMOTag-PIN fusion proteins was performed as described above. Fractions eluted with buffer $\mathrm{C}$ (containing imidazole at high concentration) were combined and dialysed overnight at $4{ }^{\circ} \mathrm{C}$ against 21 of buffer $\mathrm{A}$ in the presence of $6 \times$ His-SUMO protease. After dialysis, the protein mixture was subjected to the second round of purification on a His-Trap FF Crude column, encompassing the collection of purified PIN (lacking $6 \times$ His-SUMOTag) in the flowthrough (in buffer A) and elution of $6 \times$ His-SUMOTag together with $6 \times$ His-SUMO protease in buffer $\mathrm{D}(20 \mathrm{mM}$ Tris- $\mathrm{HCl} \mathrm{pH} 8.0,1 \mathrm{M} \mathrm{NaCl}, 600 \mathrm{mM}$ imidazole, $10 \mathrm{mM}$ 2-mercaptoethanol). PIN versions thus obtained were further purified by size-exclusion chromatography on a Superdex $7510 / 300$ GL column (GE Healthcare) and stored at $-80{ }^{\circ} \mathrm{C}$ in the presence of $30 \%$ glycerol.

\section{Substrate preparation for biochemical assays}

The $17-\mathrm{A}_{14}$ oligoribonucleotide (Invitrogen) was purified on a $10 \%$ denaturing polyacrylamide gel. Gel bands containing the oligoribonucleotide were detected by ultraviolet shadowing; they 
were then excised and the RNAs were eluted overnight at $25^{\circ} \mathrm{C}$ in an equal volume of $100 \mathrm{mM}$ Tris- $\mathrm{HCl} \mathrm{pH} 8.0,12.5 \mathrm{mM}$ EDTA, $150 \mathrm{mM} \mathrm{NaCl}, 1 \%$ SDS and phenol.

5 ' ends labelling was performed with T4 polynucleotide kinase (NEB) and $\left[\gamma^{-32} \mathrm{P}\right] \mathrm{ATP}$ (GE Healthcare) in accordance with the manufacturer's instructions.

3' ends labelling was performed with home-made $\left[{ }^{32} \mathrm{P}\right] \mathrm{pCp}$ (prepared by incubating cytidine 3'monophosphate with $\left[\gamma^{32} \mathrm{P}\right] \mathrm{ATP}$ in the presence of T4 polynucleotide kinase) and T4 RNA ligase (NEB).

Circular 17- $\mathrm{A}_{14}$ substrate was prepared by incubating $150 \mathrm{pmol}$ of the oligoribonucleotide with 250 pmol of unlabelled ATP and 4 pmol of $\left[\gamma^{32} \mathrm{P}\right] \mathrm{ATP}$ in $50 \mathrm{mM}$ Tris- $\mathrm{HCl} \mathrm{pH} 8.0,10 \mathrm{mM} \mathrm{MgCl}{ }_{2}$, $10 \mathrm{mM}$ dithiothreitol, using T4 polynucleotide kinase and T4 RNA ligase.

5'-ETS substrate was prepared with pAD268 plasmid (see Supplementary Table 1), digested with a combination of SapI and XhoI, as template for in vitro transcription, which was performed with T7 RNA polymerase (NEB) in the presence of $\left[\alpha-{ }^{32} \mathrm{P}\right] \mathrm{UTP}$ (Amersham Biosciences), in accordance with the manufacturer's instructions.

All RNA substrates were purified from 6-20\% denaturing polyacrylamide gels.

\section{Exosome co-precipitation}

Dis3-TEV-PA-associated complexes from $400 \mathrm{ml}$ of BSY1865[pBS3269-78] culture at $D_{600}=1$ were affinity-purified on IgG-Sepharose resin, followed by TEV cleavage as described previously ${ }^{25}$. Input fraction $(0.3 \mathrm{ml})$, or $7.5 \mathrm{ml}$ of the eluates, was separated by gradient SDS-PAGE, and transferred to a nitrocellulose membrane for western blot analyses. A rabbit antibody recognizing the calmodulin-binding peptide tag and cross-reacting with the Protein A tag (anti-TAP antibody; Open Biosystems) was used at a dilution of 1:2,000, followed by incubation with a goat anti-rabbithorseradish peroxidase IgG secondary antibody (Pierce) at a dilution of 1:5,000. Signals generated by enhanced chemiluminescence (ECL; GE Healthcare) were detected with a Las3000 device (Fuji).

\section{Northern blots and sequencing}

Total RNA was extracted with a standard phenol procedure ${ }^{26}$. RNA (3-10 mg) was fractionated by electrophoresis on a $6 \%$ polyacrylamide-urea gel before transfer to a Hybond $\mathrm{N}^{+}$membrane (GE Healthcare). The membrane was saturated and hybridizations were performed in Church buffer, as described previously $y^{26}$, at $42{ }^{\circ} \mathrm{C}$ for ${ }^{32} \mathrm{P}$-labelled oligonucleotide probes or at $55{ }^{\circ} \mathrm{C}$ for randomprimed PCR probes. OBS2998, OBS2999 and OBS3081 oligonucleotides hybridized in 5'-ETS. CS5 hybridized in the 5.8S rRNA ${ }^{27}$. The PCR probes against scR1, 5'-ETS and NEL025 CUT were amplified from yeast genomic DNA with oligonucleotides FWSCR1D-R, OBS2616-CS18 and PP24 , respectively. The three probes were labelled with $\left[\alpha_{-}{ }^{32} \mathrm{P}\right] \mathrm{dCTP}$ by using the MegaPrime DNA labelling system (GE Healthcare). Signals were detected by phosphorimaging on a Storm device (GE Healthcare). 


\section{References}

1. Mitchell, P. et al. The exosome: a conserved eukaryotic RNA processing complex containing multiple 3' $\rightarrow 5$ ' exoribonucleases. Cell 91, 457-466 (1997).

2. Allmang, C. et al. Functions of the exosome in rRNA, snoRNA and snRNA synthesis. EMBO J. 18, 53995410 (1999).

3. Anderson, J. S. \& Parker, R. P. The 3' to 5' degradation of yeast mRNAs is a general mechanism for mRNA turnover that requires the SKI2 DEVH box protein and 3 ' to 5' exonucleases of the exosome complex. EMBO J. 17, 1497-1506 (1998).

4. Isken, O. \& Maquat, L. E. Quality control of eukaryotic mRNA: safeguarding cells from abnormal mRNA function. Genes Dev. 21, 1833-1856 (2007).

5. Wyers, F. et al. Cryptic pol II transcripts are degraded by a nuclear quality control pathway involving a new poly(A) polymerase. Cell 121, 725-737 (2005).

6. Lebreton, A. \& Séraphin, B. Exosome-mediated quality control: Substrate recruitment and molecular activity. Biochim. Biophys. Acta 1779, 558-565 (2008).

7. Dziembowski, A., Lorentzen, E., Conti, E. \& Séraphin, B. A single subunit, Dis3, is essentially responsible for yeast exosome core activity. Nature Struct. Mol. Biol. 14, 15-22 (2007).

8. Liu, Q., Greimann, J. C. \& Lima, C. D. Reconstitution, activities, and structure of the eukaryotic RNA exosome. Erratum. Cell 131, 188189 (2007).

9. Liu, Q., Greimann, J. C. \& Lima, C. D. Reconstitution, activities, and structure of the eukaryotic RNA exosome. Cell 127, 1223-1237 (2006).

10. Hernandez, H. et al. Subunit architecture of multimeric complexes isolated directly from cells. EMBO Rep. 7, 605-610 (2006).

11. Frazao, C. et al. Unravelling the dynamics of RNA degradation by ribonuclease II and its RNA-bound complex. Nature 443, 110-114 (2006).

12. Lorentzen, E. et al. Structure of the active subunit of the yeast exosome core, Rrp44: diverse modes of substrate recruitment in the RNase II nuclease family. Mol. Cell 29, 717-728 (2008).

13. Zuo, Y. et al. Structural basis for processivity and single-strand specificity of RNase II. Mol. Cell 24, 149-156 (2006).

14. Allmang, C. et al. The yeast exosome and human PM-Scl are related complexes of $3 \rightarrow 5$, exonucleases. Genes Dev. 13, 2148-2158 (1999).

15. Arcus, V. L. et al. Distant structural homology leads to the functional characterization of an archaeal PIN domain as an exonuclease. J. Biol. Chem. 279, 16471-16478 (2004).
16. Glavan, F., Behm-Ansmant, I., Izaurralde, E. \& Conti, E. Structures of the PIN domains of SMG6 and SMG5 reveal a nuclease within the mRNA surveillance complex. EMBO J. 25, 5117-5125 (2006).

17. Belli, G. et al. An activator/repressor dual system allows tight tetracycline-regulated gene expression in budding yeast. Nucleic Acids Res. 26, 942-947 (1998).

18. Couttet, P. et al. Messenger RNA deadenylylation precedes decapping in mammalian cells. Proc. Natl Acad. Sci. USA 94, 5628-5633 (1997).

19. Mandl, C. W., Heinz, F. X., Puchhammer-Stockl, E. \& Kunz, C. Sequencing the termini of capped viral RNA by 5'-3' ligation and PCR. Biotechniques 10 , 484-486 (1991).

20. Kadaba, S. et al. Nuclear surveillance and degradation of hypomodified initiator tRNAMet in S. cerevisiae. Genes Dev. 18, 1227-1240 (2004).

21. LaCava, J. et al. RNA degradation by the exosome is promoted by a nuclear polyadenylation complex. Cell 121, 713-724 (2005).

22. Vanacova, S. et al. A new yeast poly(A) polymerase complex involved in RNA quality control. PLoS Biol. 3, e189 (2005).

23. Carpousis, A. J. The RNA degradosome of Escherichia coli: an mRNA-degrading machine assembled on RNase E. Annu. Rev. Microbiol. 61, 71-87 (2007).

24. Bonneaud, N. et al. A family of low and high copy replicative, integrative and single-stranded $\mathrm{S}$. cerevisiae/E. coli shuttle vectors. Yeast 7, 609-615 (1991).

25. Rigaut, G. et al. A generic protein purification method for protein complex characterization and proteome exploration. Nature Biotechnol. 17, 10301032 (1999).

26. Daugeron, M. C., Mauxion, F. \& Séraphin, B. The yeast POP2 gene encodes a nuclease involved in mRNA deadenylation. Nucleic Acids Res. 29, 24482455 (2001).

27. Saveanu, C. et al. Nog2p, a putative GTPase associated with pre-60S subunits and required for late 60S maturation steps. EMBO J. 20, 6475-6484 (2001). 